\title{
Performance Analysis of Multiband OFDM for UWB Communication
}

\author{
Chris Snow, Lutz Lampe, and Robert Schober \\ Department of Electrical \& Computer Engineering \\ University of British Columbia, Vancouver, Canada \\ \{csnow,lampe,rschober\}@ece.ubc.ca
}

\begin{abstract}
The frequency-hopping orthogonal frequencydivision multiplexing (OFDM) proposal, known as Multiband OFDM, is a strong contender for the physical layer IEEE standard for high-rate wireless personal area networks (WPANs) based on ultra-wideband (UWB) transmission. In this paper, we analyze the performance of the Multiband OFDM proposal. To this end, we (a) study the channel model developed under IEEE 802.15 for UWB radio from a frequency-domain perspective suited for OFDM transmission, (b) develop and quantify the appropriate information-theoretic performance measures, (c) compare these measures with simulation results for Multiband OFDM systems, and (d) consider the influence of practical, imperfect channel estimation on the performance.
\end{abstract}

\section{INTRODUCTION}

Ultra-wideband (UWB) radio has recently been popularized as a technology for short-range, high data rate communication and locationing applications (cf. e.g. [1]). While there exist several forms of UWB radio, the research literature to date has focused almost exclusively on impulse radio [2]. Recently, the IEEE 802.15 wireless personal area networks (WPANs) standardization group has organized task group 3a to develop an alternative physical layer based on UWB signaling [3]. Currently there are two main contenders for this standard: a frequency-hopping orthogonal frequency-division multiplexing (OFDM) proposal known as Multiband OFDM and a codedivision multiple access (CDMA) based technique.

In this paper, we consider the proposed Multiband OFDM standard [4]. Multiband OFDM is a conventional OFDM system [5] combined with bit-interleaved coded modulation (BICM) [6] for error prevention and frequency hopping for improved diversity and multiple access. The signal bandwidth is $528 \mathrm{MHz}$, which makes it a UWB signal according to the definition by the US Federal Communications Commission (FCC) [1], and hopping between three adjacent frequency bands is employed for first generation devices [4]. Thus, the Multiband OFDM proposal is a rather pragmatic approach for UWB transmission, which builds upon the proven BICMOFDM concept. ${ }^{1}$

The objective of this paper is to study the suitability and to analyze the (potential) performance of Multiband OFDM for UWB transmission. Thereby, our investigations rely on the new UWB channel model developed under IEEE 802.15 [7]. We analyze this channel model in the frequency domain and

\footnotetext{
${ }^{1}$ Throughout this paper, the term "Multiband OFDM" refers to the particular standard proposal [4], whereas "BICM-OFDM" referes to the general concept of combining BICM and OFDM.
}

extract the relevant statistical parameters that mainly determine the performance of OFDM based transmission. In particular, the amount of diversity available in the wireless channel as a function of the signal bandwidth is examined. As appropriate performance measures for coded communication systems, we discuss the capacity and cutoff rate limits of BICM-OFDM systems on UWB channels. In this context, since one limiting factor of performance in practical and especially in wideband BICM-OFDM systems is the availability of high-quality channel state estimates, the effect of imperfect channel state information (CSI) at the receiver is specifically addressed. Furthermore, the information-theoretic performance limits are compared with simulated bit-error rate (BER) results for the proposed Multiband OFDM [4].

This paper is organized as follows. Section II describes the Multiband OFDM system and the UWB channel model under consideration. The properties of the UWB channel relevant for OFDM transmission are examined in Section III. Section IV presents the capacity and cutoff rate analysis together with numerical results for perfect and imperfect CSI. Simulation results for the Multiband OFDM system are presented and compared with the theoretical benchmark measures in Section V, and conclusions are given in Section VI.

\section{Multiband OFDM System And UWB Channel}

In this section, the Multiband OFDM transmission system and channel model are introduced in necessary detail. The transmitter processing is performed according to the standard proposal [4], for the receiver we propose a conventional stateof-the art architecture including channel estimation based on pilot symbols.

\section{A. Multiband OFDM Transmitter}

The block diagram of the Multiband OFDM transmitter is shown in Fig 1a). Classical BICM [6] with a punctured maximum free distance rate $1 / 3$ constraint length 7 convolutional encoder is applied. The interleaved coded bits are mapped to quaternary phase-shift keying (QPSK) symbols using Gray labeling. The QPSK symbols are optionally repeated in time and/or frequency and grouped to form OFDM symbols with $N=128$ tones (or subcarriers). Via inverse fast Fourier transform (IFFT) the time domain signal is generated and a cyclic prefix of 32 symbols is appended. The radio frequency (RF) transmit signal hops after each OFDM symbol between 


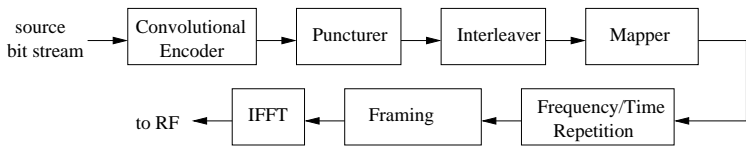

(a) Transmitter

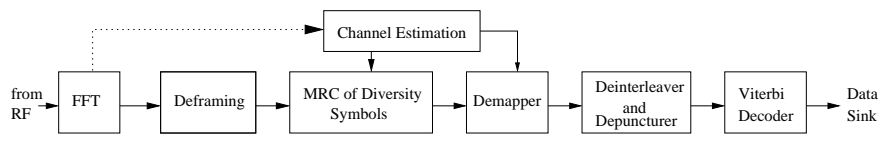

(b) Receiver

Fig. 1. Block diagram of Multiband OFDM transmission system.

three $528 \mathrm{MHz}$ frequency bands with center frequencies at $3.432,3.960$, and $4.448 \mathrm{GHz}$ (see [4] for more details).

The Multiband OFDM transmission is organized in packets with varying payload sizes. For our purposes it suffices to say that the standard proposal [4] envisages the transmission of two pilot OFDM symbols (all tones are pilots) per frequency band in the packet header for channel estimation.

\section{B. UWB Channel Model}

For a meaningful performance analysis of the Multiband OFDM proposal, we consider the channel model developed under IEEE 802.15 for UWB systems [7]. The channel impulse response is a version of the Saleh-Valenzuela model [8] modified to fit the properties of measured UWB channels. Multipath rays arrive in clusters, with exponentially distributed cluster and ray interarrival times. Both clusters and rays have decay factors chosen to meet a given power decay profile. The ray amplitudes are modeled as lognormal random variables, and each cluster of rays also undergoes a lognormal fading. To provide a fair system comparison, the total multipath energy is normalized to unity. Finally, the entire impulse response undergoes an "outer" lognormal shadowing. The channel impulse response is assumed time invariant during the transmission period of (at least) one packet (see [7] for a detailed description).

Four separate channel models (CM1-CM4) have been made available for UWB system modeling, each with arrival rates and decay factors chosen to match a different usage scenario. The four models are tuned to fit 0-4 m Line-of-Sight (LOS), 0-4 m non-LOS, 4-10 m non-LOS, and an "extreme nonLOS multipath channel", respectively. The means and standard deviations of the outer lognormal shadowing are the same for all four models. The model parameters can be found in [7, Table 2].

\section{Multiband OFDM Receiver}

The block diagram of the receiver considered in this paper is depicted in Fig 1b). We assume perfect timing and frequency synchronization. Furthermore, for the system parameters and UWB channel model outlined above, the cyclic prefix can safely be assumed longer than the delay spread of the channel impulse response. Thus, after FFT we see an equivalent $N$ dimensional frequency non-selective vector channel, expressed as [5],

$$
\boldsymbol{Y}[k]=\boldsymbol{X}_{d}[k] \boldsymbol{H}+\boldsymbol{N}[k],
$$

where the vector notation $\boldsymbol{Z}[k]=\left[Z_{1}[k] \ldots Z_{N}[k]\right]^{T}$ is used ( ${ }^{T}$ denotes transpose) and $\boldsymbol{X}_{d}[k]$ is the $N \times N$ diagonal matrix with elements $X_{i}[k]$ at its main diagonal. $Y_{i}[k], X_{i}[k]$, and $N_{i}[k]$ are the received symbol, the transmitted QPSK symbol, and the additive white Gaussian noise (AWGN) sample on frequency tone $i=1 \ldots N$ of the $k$ th OFDM symbol, respectively. The vector $\boldsymbol{H}$ contains the frequency domain samples of the channel transfer function on tones $i=1 \ldots N$ and is assumed constant over the considered time span (see Section II-B).

The channel estimation, diversity combining, demapping, and decoding are briefly described in the following.

1) Channel Estimation: We implement a least-squares error (LSE) channel estimator for the time-domain channel impulse response (CIR) using the $P$ pilot OFDM symbols in the packet header. For a more general treatment, we let $P$ be a design parameter, but we note that $P=2$ is proposed in [4]. The responses in different frequency bands can be estimated seperately, since pilot symbols are transmitted for each band. The LSE estimator is chosen instead of minimummean-square error (MMSE) estimation because it does not require assumptions regarding the statistical structure of the channel correlations. Furthermore, it has been shown that LSE and MMSE estimation perform almost equally well for cases of practical interest [9].

The LSE estimator exploits the fact that the CIR has a maximum of $L \leq N$ taps. Starting from (1), the frequencydomain vector channel estimate is straightforwardly obtained as (cf. e.g. [9])

$$
\hat{\boldsymbol{H}}=\boldsymbol{H}+\boldsymbol{E},
$$

where the channel estimation error vector $\left(.{ }^{\dagger}\right.$ denotes Hermitian transpose)

$$
\boldsymbol{E}=\boldsymbol{F}_{N \times L} \boldsymbol{F}_{N \times L}^{\dagger} \cdot \frac{1}{P} \sum_{k=1}^{P} \boldsymbol{X}_{d}^{\dagger}[k] \boldsymbol{N}[k]
$$

is independent of $\boldsymbol{H}$ and zero-mean Gaussian distributed with correlation matrix

$$
\begin{aligned}
\boldsymbol{R}_{\boldsymbol{E} \boldsymbol{E}} & =\boldsymbol{F}_{N \times L} \boldsymbol{F}_{N \times L}^{\dagger}\left(\frac{\sigma_{N}^{2}}{P^{2}} \sum_{k=1}^{P} \boldsymbol{X}_{d}^{\dagger}[k] \boldsymbol{X}_{d}[k]\right) \boldsymbol{F}_{N \times L} \boldsymbol{F}_{N \times L}^{\dagger} \\
& =\boldsymbol{F}_{N \times L} \boldsymbol{F}_{N \times L}^{\dagger} \sigma_{N}^{2} / P
\end{aligned}
$$

In (3) and (4), $\boldsymbol{F}_{N \times L}$ denotes the normalized $N \times L$ FFT matrix with elements $\mathrm{e}^{-\mathrm{j} \mu \nu 2 \pi / N} / \sqrt{N}$ in row $\mu$ and column $\nu$, and $\sigma_{N}^{2}$ is the AWGN variance. For the last step in (4) we assumed the use of constant modulus pilot symbols $\left|X_{i}[k]\right|=$ 1. We observe from (2) and (4) that the LSE channel estimate is disturbed by correlated Gaussian noise with variance

$$
\sigma_{E}^{2}=\frac{L}{N P} \sigma_{N}^{2}=\eta \sigma_{N}^{2}
$$


In order to keep complexity low we do not attempt to exploit the correlation, and we further assume that because of interleaving the effect of correlation is negligible. We will refer to parameter $\eta=L /(N P)$ in (5) when evaluating the performance of Multiband OFDM with imperfect CSI in Sections IV-C and V.

2) Diversity Combining, Demapping, and Decoding: Maximum-ratio combining (MRC) [10] in case of time and/or frequency spreading (see Section II-A and [4]) and demapping in the standard BICM fashion [6] are performed based on the channel estimator output $\hat{\boldsymbol{H}}$. The resulting "soft" bit metrics are deinterleaved and depunctured, and a soft-input Viterbi decoder is used to restore the original bitstream.

\section{UWB ChANNEL AND DIVERSITY ANALYSIS FOR MulTibAND OFDM SYSTEMS}

The UWB channel model developed under IEEE 802.15 [7] (see Section II-B) is a stochastic time-domain model. In this section, we consider a stochastic frequency-domain description, i.e., we include transmitter IFFT and receiver FFT into the channel definition and consider realizations of $\boldsymbol{H}$ in (1). In doing so, we intend to (a) extract the channel parameters relevant for the performance of OFDM-based UWB systems, (b) examine whether the Multiband OFDM proposal is adequate to exploit the channel characteristics, (c) quantify the impact of the different UWB channel types CM1-CM4 on system performance, and (d) possibly enable a classification of the UWB channel model into more standard channel models used in communication theory.

From (1) we observe that the OFDM transmit signal experiences a frequency non-selective fading channel with fading along the frequency axis. Thereby, the outer lognormal shadowing term is irrelevant for the fading characteristics as it affects all tones equally. Hence, the lognormal shadowing term is omitted in the following considerations. Denoting the lognormal term by $G$, we obtain the corresponding normalized frequency-domain fading coefficients by

$$
H_{i}^{\mathrm{n}}=H_{i} / G \text {. }
$$

\section{A. Marginal Distribution}

The first parameter of interest is the marginal distribution of $H_{i}^{\mathrm{n}}$, i.e., the probability density function (pdf) $p\left(H_{i}^{\mathrm{n}}\right)$.

First, we note that the frequency-domain coefficient $H_{i}^{\mathrm{n}}$ is a zero mean random variable since the time-domain multipath components are zero mean quantities. Furthermore, we have observed that $H_{i}^{\mathrm{n}}$ is, in good approximation, circularly symmetric complex Gaussian distributed, which is explained by the fact that $H_{i}^{\mathrm{n}}$ results from the superposition of relatively many time-domain multipath components. Since these multipath components are mutually statistically independent, the variance of $H_{i}^{\mathrm{n}}$ is independent of the tone index $i$.

Figure 2 shows experimental measurements of the pdfs $p\left(\left|H_{i}^{\mathrm{n}}\right|\right)$ of the magnitude frequency-domain gain $\left|H_{i}^{\mathrm{n}}\right|$ for the different channel models CM1-CM4. As can be seen, the experimental distributions agree well with the exact Rayleigh

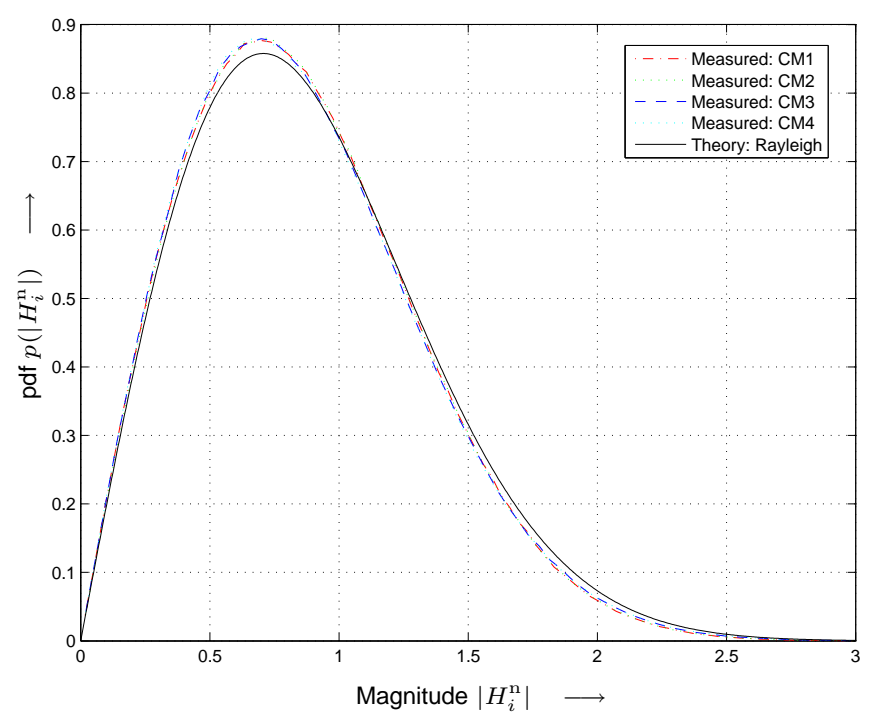

Fig. 2. Distributions of normalized channel magnitude $\left|H_{i}^{\mathrm{n}}\right|$ for channel types CM1-CM4. For comparison: Rayleigh distribution with same variance.

distribution of equal variance, which is in accordance with the statements above.

\section{B. Correlation}

The findings in the previous section indicate that the OFDM signal effectively experiences a (classical) frequency nonselective Rayleigh fading channel. Therefore, knowledge of the second-order channel statistics, i.e., the correlation between different fading coefficients $H_{i}^{\mathrm{n}}$ and $H_{j}^{\mathrm{n}}, i \neq j$, is important for the design and assessment of diversity techniques such as coding, interleaving, and frequency hopping, which are envisioned in the Multiband OFDM system. Since coding is performed over all bands, we treat all 3 bands jointly.

As an appropriate figure of merit we examine the ordered eigenvalues of the $3 \cdot N \times 3 \cdot N$ correlation matrix $\boldsymbol{R}_{\boldsymbol{H}^{\mathrm{n}}} \boldsymbol{H}^{\mathrm{n}}$ of $\boldsymbol{H}^{\mathrm{n}}=\left[H_{1}^{\mathrm{n}} \ldots H_{3 \cdot N}^{\mathrm{n}}\right]^{T}$. Figure 3 shows the first 40 ordered eigenvalues (every second from 1st to 21st, and the 30th and 40th) of the measured $\boldsymbol{R}_{\boldsymbol{H}^{\mathrm{n}} \boldsymbol{H}^{\mathrm{n}}}$, which has been obtained from averaging over 10000 channel realizations, as a function of the total employed signal bandwidth. We only show results for channel models CM1 and CM4, which constitute the two extreme cases as the corresponding impulse responses have the least (CM1) and most (CM4) independent multipath components. The respective curves for models CM2 and CM3 lie in between those for CM1 and CM4.

From Figure 3 we infer the following conclusions:

1) By increasing the bandwidth of the OFDM signal the diversity order of the equivalent frequency-domain channel, i.e., the number of the significant non-zero

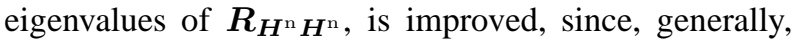
more time-domain multipath components are resolved. However, a $1500 \mathrm{MHz}$ total bandwidth provides already $\geq 40$ (CM4) and $\geq 30$ (CM1) strong diversity branches. This indicates that the $528 \mathrm{MHz}$ bandwidth and 3-band frequency hopping of Multiband OFDM is a favorable 


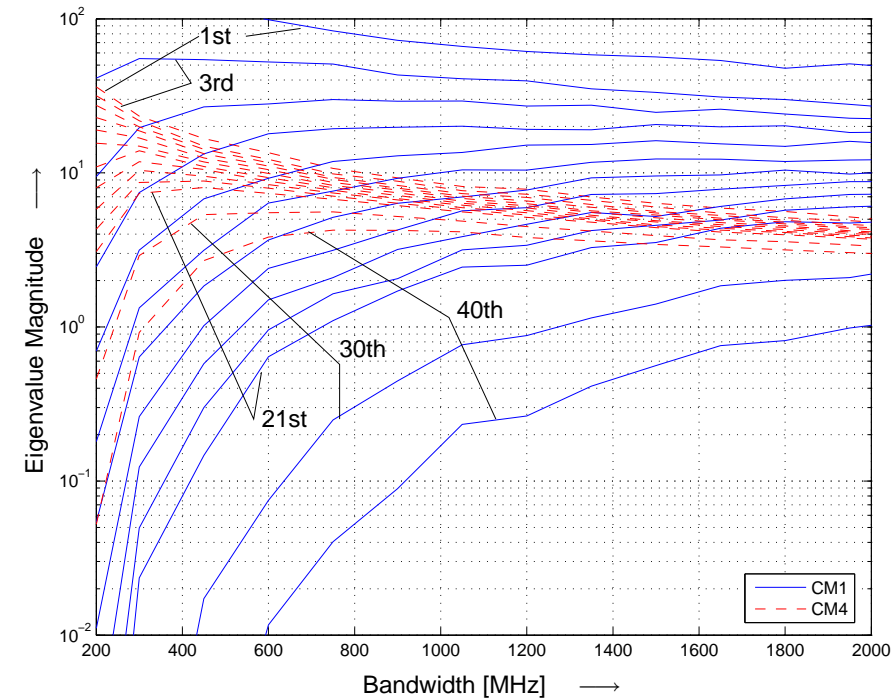

Fig. 3. First 40 ordered eigenvalues of the correlation matrix $\boldsymbol{R}_{\boldsymbol{H}}^{\mathrm{n}} \boldsymbol{H}^{\mathrm{n}}$ (every second from 1 st to 21 st, and the 30 th and 40 th).

compromise between complexity and available diversity for CM1.

2) Since the system, comprising the convolutional code (see Section II-A) with free distance $\leq 15$ (depending on the puncturing) and spreading factor 1,2 , and 4, can at best exploit diversities of order 15, 30 and 60, respectively, bandwidths of more than $500 \mathrm{MHz}$ per band would only be beneficial for the lowest data-rate modes, and then only for very low error rates.

3) Though CM4 provides higher diversity order than CM1, the latter appears advantageous for high data-rate modes with code puncturing due to its larger first ordered eigenvalues.

In summary, we conclude that, given a particular realization of the lognormal shadowing term, the equivalent frequencydomain channel $\boldsymbol{H}$ in (1) is well approximated by a Rayleigh fading channel with relatively high "fading rate", which increases from CM1 to CM4.

\section{CAPACITy AND Cutoff Rate AnAlysis}

The purpose of this section is to quantify potential data rates and power efficiencies of OFDM-based UWB transmission. Of particular interest here are (a) the channel capacity and cutoff rate, which are widely accepted performance measures for coded transmission, (b) the influence of the particular channel model (CM1-CM4), and (c) the effect of imperfect channel estimation on these measures. Since coding and interleaving are limited to single realizations of lognormal shadowing, we focus on the notion of outage probability, i.e., the probability that the instantaneous capacity and cutoff rate for a given channel realization $\boldsymbol{H}$ fall below a certain threshold. These theoretical performance measures will be compared with simulation results for the Multiband OFDM system in Section $\mathrm{V}$.

In Section IV-A, we briefly review the capacity and cutoff rate expressions for the considered BICM-OFDM system. The required conditional pdf of the channel output is given in Section IV-B. Finally, numerical results are presented and discussed in Section IV-C.

\section{A. Capacity and Cutoff Rate Expressions}

The instantaneous capacity in bits per complex dimension of an $N$ tone BICM-OFDM system is given by (cf. e.g. [6], [11])

$$
C(\boldsymbol{H})=m-\frac{1}{N} \sum_{\ell=1}^{m} \sum_{i=1}^{N} \mathrm{E}_{b, Y_{i}}\left\{\log _{2}\left(\frac{\sum_{X_{i} \in \mathcal{X}} p\left(Y_{i} \mid \hat{H}_{i}, X_{i}\right)}{\sum_{X_{i} \in \mathcal{X}_{b}^{\ell}} p\left(Y_{i} \mid \hat{H}_{i}, X_{i}\right)}\right)\right\} .
$$

In (7), $m$ is the number of bits per symbol, $\mathcal{X}$ is the signal constellation and $\mathcal{X}_{b}^{\ell}$ is the set of all constellation points $X \in \mathcal{X}$ whose label has the value $b \in\{0,1\}$ in position $\ell$, $p\left(Y_{i} \mid \hat{H}_{i}, X_{i}\right)$ is the pdf of the channel output $Y_{i}$ for given input $X_{i}$ and channel estimate $\hat{H}_{i}$, and $\mathrm{E}_{z}\{\cdot\}$ denotes expectation with respect to $z$. For Multiband OFDM, $\mathcal{X}$ is the QPSK signal constellation and $m=2$ is valid.

Similarly, we can express the instantaneous cutoff rate in bits per complex dimension as (cf. e.g. [6], [11])

$$
R_{0}(\boldsymbol{H})=m\left(1-\log _{2}(B(\boldsymbol{H})+1)\right)
$$

with the instantaneous Bhattacharya parameter $(\bar{b}$ denotes the complement of $b$ )

$$
B(\boldsymbol{H})=\frac{1}{m N} \sum_{\ell=1}^{m} \sum_{i=1}^{N} \mathrm{E}_{b, Y_{i}}\left\{\sqrt{\frac{\sum_{X_{i} \in \mathcal{X}_{\bar{b}}^{\ell}} p\left(Y_{i} \mid \hat{H}_{i}, X_{i}\right)}{\sum_{X_{i} \in \mathcal{X}_{b}^{\ell}} p\left(Y_{i} \mid \hat{H}_{i}, X_{i}\right)}}\right\} .
$$

\section{B. Conditional PDF}

In order to calculate capacity and cutoff rate, we require the conditional pdf $p\left(Y_{i} \mid \hat{H}_{i}, X_{i}\right)$. In the case of perfect CSI we have $\hat{H}_{i}=H_{i}$, and $p\left(Y_{i} \mid \hat{H}_{i}, X_{i}\right)$ is a Gaussian pdf with mean $H_{i} X_{i}$ and variance $\sigma_{N}^{2}$.

To obtain $p\left(Y_{i} \mid \hat{H}_{i}, X_{i}\right)$ for the more realistic case of imperfect CSI, we assume the application of LSE channel estimation as described in Section II-C. According to the results of Section III-A and since channel estimation is performed for one realization $G$ of the lognormal shadowing term, we further assume zero-mean circularly symmetric Gaussian distributed channel coefficients $H_{i}$ with variance $\sigma_{H}^{2}=G^{2}$ (see Eq. (6)). This means that $\hat{H}_{i}$ is also zero-mean Gaussian distributed with variance $\sigma_{\hat{H}}^{2}=\sigma_{H}^{2}+\sigma_{E}^{2}$ (see Eqs. (2) and (5)). Let $\mu$ be the correlation between $H_{i}$ and $\hat{H}_{i}$,

$$
\mu=\frac{\mathrm{E}_{H_{i}, \hat{H}_{i}}\left\{H_{i} \hat{H}_{i}^{\dagger}\right\}}{\sigma_{H} \sigma_{\hat{H}}}=\sqrt{\frac{\sigma_{H}^{2}}{\sigma_{E}^{2}+\sigma_{H}^{2}}}=\sqrt{\frac{\gamma}{\gamma+\eta}},
$$

where $\eta$ is defined in (5) and $\gamma=\sigma_{H}^{2} / \sigma_{N}^{2}$ is the signal-to-noise ratio (SNR). Then, we can arrive via algebraic manipulations at (cf. e.g. [12])

$$
p\left(Y_{i} \mid \hat{H}_{i}, X_{i}\right)=\frac{1}{\pi\left(\sigma_{N}^{2}\left(\eta \mu^{2}+1\right)\right)} \exp \left(-\frac{\left|Y_{i}-X_{i} \hat{H}_{i} \mu^{2}\right|^{2}}{\sigma_{N}^{2}\left(\eta \mu^{2}+1\right)}\right) \text {. }
$$




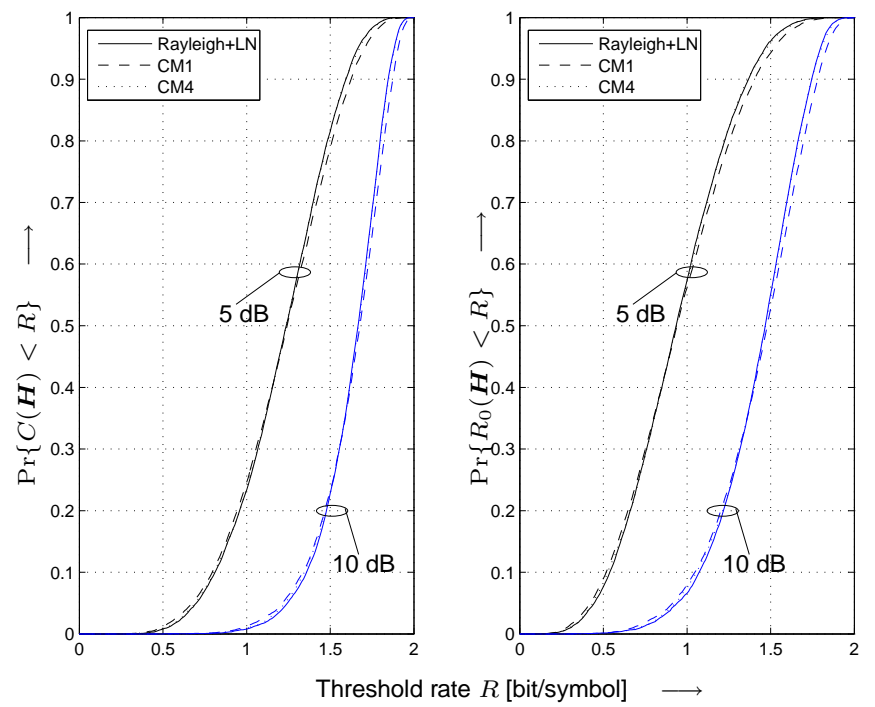

Fig. 4. Outage probability for $10 \log _{10}\left(\bar{E}_{s} / \mathcal{N}_{0}\right)=5 \mathrm{~dB}$ and $10 \mathrm{~dB}$ and perfect CSI. Left: Outage capacity. Right: Outage cutoff rate.

The Gaussian density of (11) implies that the system with imperfect CSI can be seen as a system with perfect CSI at an equivalent SNR of

$$
\gamma_{e}=\frac{\mathrm{E}_{\hat{H}_{i}}\left\{\left|\hat{H}_{i}\right|^{2}\right\} \mu^{4}}{\sigma_{N}^{2}\left(\eta \mu^{2}+1\right)}=\frac{\gamma}{\eta\left(1+\frac{1}{\gamma}\right)+1} .
$$

We note that in the high SNR regime the loss due to estimation error reaches a constant value of $1 /(\eta+1)$.

\section{Numerical Capacity and Cutoff Rate Results}

We evaluated expressions (7) and (8) via Monte Carlo simulation using 10000 realizations of each UWB channel model CM1-CM4. To keep the figures legible, we present representative results for CM1 and CM4 only. The CM2 and CM3 models not shown have performance figures that lie in between those of CM1 and CM4 (cf. also Section III-B). For comparison we also include results for independent and identically distributed (i.i.d.) Rayleigh fading on each tone and an outer lognormal shadowing term identical to that of the UWB models (labeled as "Rayleigh + LN").

1) Perfect CSI: First, we consider the case of perfect CSI. Figure 4 shows the outage capacity $\operatorname{Pr}\{C(\boldsymbol{H})<R$ ) (left) and cutoff rate $\operatorname{Pr}\left\{R_{0}(\boldsymbol{H})<R\right)$ (right) as a function of the threshold rate $R$ for $10 \log _{10}\left(\bar{E}_{s} / \mathcal{N}_{0}\right)=5 \mathrm{~dB}$ and $10 \mathrm{~dB}$, respectively, where $\bar{E}_{s}$ is the average received energy per symbol and $\mathcal{N}_{0}$ denotes the two-sided power spectral density of the complex noise.

It can be seen that both capacity and cutoff rate for the UWB channel models are similar to the respective parameters of an i.i.d. Rayleigh fading channel with additional lognormal shadowing. In fact, the curves for CM4, which provides the highest diversity (see Section III-B), are essentially identical to those for the idealized i.i.d. model. The high diversity provided by the UWB channel also results in relatively steep outage curves, which means that transmission reliability can

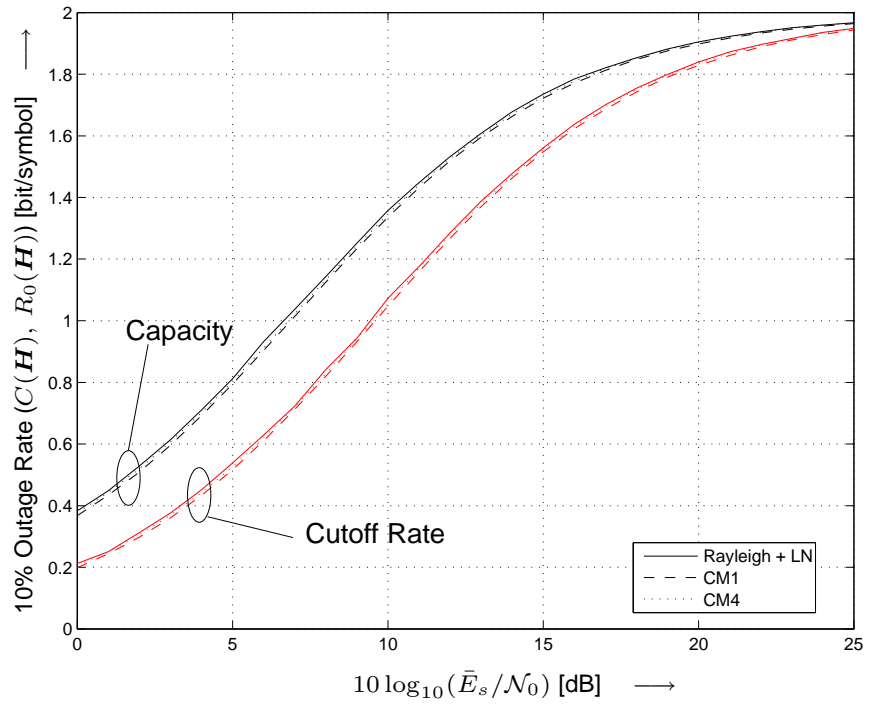

Fig. 5. $10 \%$ outage capacity and cutoff rate for perfect CSI.

be considerably improved by deliberately introducing coding redundancy. This effect is a little more pronounced for the capacity measure relevant for more powerful coding. On the other hand, the effect of shadowing, which cannot be averaged out by coding, causes a flattening towards low outage probabilities $\leq 0.1$. In the high outage probability range we note that CM1 is slightly superior to CM4, which is due to the large dominant eigenvalues of CM1 identified in Section III-B.

In Figure 5 we consider the $10 \%$ outage $^{2}$ capacity and cutoff rate as a function of the SNR $10 \log _{10}\left(\bar{E}_{s} / \mathcal{N}_{0}\right)$. Again we note the close similarity between the UWB channel models and the i.i.d. Rayleigh fading channel with lognormal shadowing. A comparison of the capacity with the corresponding cutoff rate curves indicates that decent gains of $2.5 \mathrm{~dB}$ to $3 \mathrm{~dB}$ in power efficiency can be anticipated by the application of more powerful capacity approaching codes instead of convolutional codes, which are propopsed in [4] and which usually perform in the vicinity of the cutoff rate.

2) Imperfect CSI: Figure 6 shows the SNR loss due to LSE channel estimation according to Eq. (12) with various values of $\eta$. For reference, the Multiband OFDM system uses $P=2$, $N=128$, and so choosing $L=32$ (equal to the cyclic prefix length) leads to $\eta=0.125$.

We can see from Figure 6 that the performance penalty due to imperfect CSI is about $0.5 \mathrm{~dB}$ in the range of interest for the Multiband OFDM system. Reducing the channel estimation overhead to $P=1(\eta=0.25)$ could be an interesting alternative for short packets, as the additional loss is only about $0.5 \mathrm{~dB}$ in $\bar{E}_{s} / \mathcal{N}_{0}$ (in terms of required energy per information bit $\bar{E}_{b}$ the loss is even smaller). Further reduction of pilot tones is not advisable as the gains in throughput are outweighed by the losses in power efficiency.

\footnotetext{
${ }^{2}$ We note that $10 \%$ outage is a typically chosen value for UWB systems and the considered channel model [4].
} 


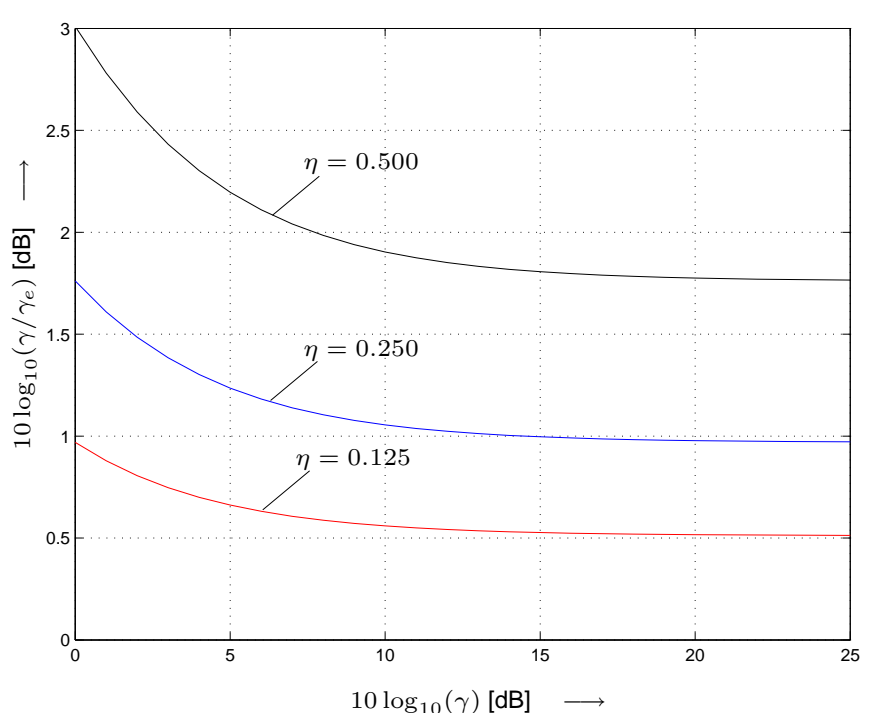

Fig. 6. Loss in SNR due to LSE channel estimation with different $\eta$ according to (12).

\section{Simulation Results}

In this section, simulation results for the Multiband OFDM system described in Section II and the UWB channel model CM1 (using 100 realizations) are presented. We consider four different transmission modes with data rates of $80,160,320$, and $480 \mathrm{Mbps}$ corresponding to $0.25,0.50,1.00$, and 1.50 bit/symbol, respectively (see [4, Table 3] for the details of puncturing etc.). In the simulations, detection is performed with perfect CSI as well as with LSE channel estimation and $\eta=0.125$. Following the procedure in [4], we report the worst-case performance for the best $90 \%$ of channel realizations, which corresponds to the $10 \%$ outage measures presented in the previous section.

Figure 7 (markers) shows the $10 \log _{10}\left(\bar{E}_{s} / \mathcal{N}_{0}\right)$ values required to achieve BER $\leq 10^{-5}$ for the $90 \%$ best channel realizations, together with the corresponding $10 \%$ outage cutoff rates. The simulated SNR points are reasonably close to the cutoff-rate curves, with some variations due to the different coding and puncturing schemes. These results (a) justify the relevance of the information-theoretic measure and (b) confirm the coding approach used in Multiband OFDM. More specifically, the diversity provided by the UWB channel is effectively exploited by the chosen convolutional coding and interleaving scheme. Furthermore, the system with LSE channel estimation performs within $0.5-0.7 \mathrm{~dB}$ of the perfect CSI case as was expected from the cutoff-rate analysis.

\section{Conclusions}

In this paper, the application of Multiband OFDM for UWB communication has been analyzed. We have shown that the UWB channel model developed under IEEE 802.15 is seen by OFDM systems as "fast" frequency non-selective Rayleigh fading with additional shadowing. The $528 \mathrm{MHz}$ signal bandwidth chosen for Multiband OFDM essentially captures the diversity provided by the UWB channel. As a result, we have found that the information-theoretic limits of

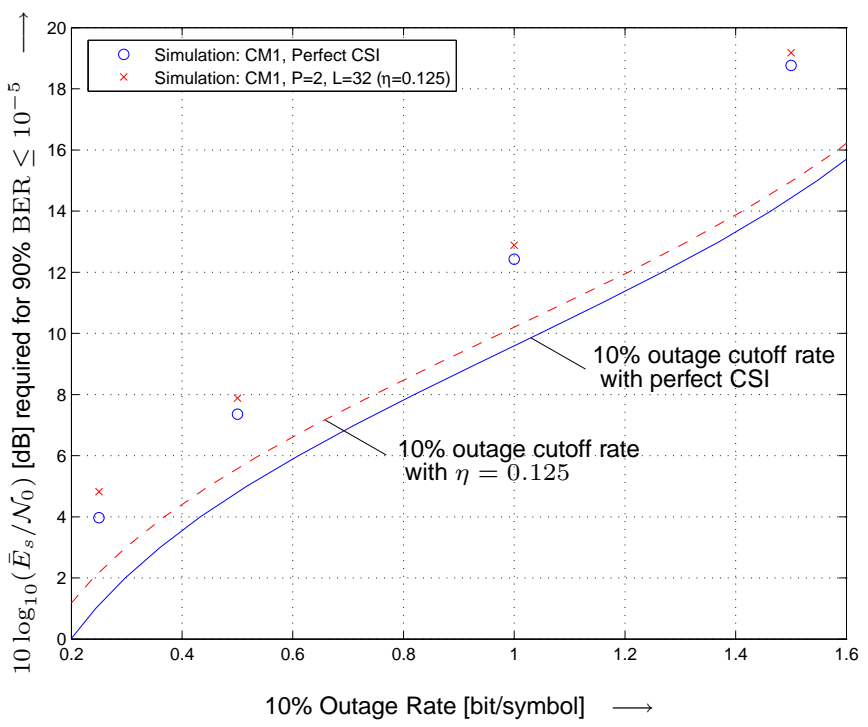

Fig. 7. $10 \log _{10}\left(\bar{E}_{s} / \mathcal{N}_{0}\right)$ required to achieve $\mathrm{BER} \leq 10^{-5}$ for the $90 \%$ best channel realizations (markers). For comparison: 10\% outage cutoff rate (lines). Channel model CM1 and LSE channel estimation.

the UWB channel are similar to those of a perfectly interleaved Rayleigh fading channel plus shadowing. The BICM-OFDM scheme proposed for Multiband OFDM performs close to the outage cutoff-rate measure and is thus well suited to exploit the available diversity. From the comparison of outage capacity and outage cutoff rate we anticipate that more powerful coding (e.g. Turbo codes) improves power efficiency by up to $3 \mathrm{~dB}$. A simple LSE channel estimator has been shown to enable performance within $0.5-0.7 \mathrm{~dB}$ of the perfect CSI case for the proposed Multiband OFDM system.

\section{REFERENCES}

[1] S. Roy, J. Foerster, V. Somayazulu, and D. Leeper, "Ultrawideband Radio Design: The Promise of High-Speed, Short-Range Wireless Connectivity," Proceedings of the IEEE, vol. 92, pp. 295-311, Feb. 2004.

[2] M. Z. Win and R. A. Scholtz, "Impulse radio: How it works," IEEE Commun. Lett., vol. 2, no. 2, pp. 36-38, Feb. 1998.

[3] "See: http://www.ieee802.org/15/pub/TG3a.html."

[4] IEEE P802.15, "Multiband OFDM Physical Layer Proposal for IEEE 802.15 Task Group 3a (Doc. Number P802.15-03/268r3)," Mar. 2004.

[5] J. Bingham, ADSL, VDSL, and Multicarrier Modulation. New York: John Wiley \& Sons, 2000.

[6] G. Caire, G. Taricco, and E. Biglieri, "Bit-Interleaved Coded Modulation," IEEE Trans. Inform. Theory, vol. 44, no. 3, pp. 927-946, May 1998.

[7] A. F. Molisch, J. R. Foerster, and M. Pendergrass, "Channel Models for Ultrawideband Personal Area Networks," IEEE Wireless Commun. Mag., pp. 14-21, Dec. 2003.

[8] A. Saleh and R. Valenzuela, "A Statistical Model for Indoor Multipath Propagation,” IEEE J. Select. Areas Commun., vol. SAC-5, no. 2, pp. 128-137, Feb. 1987.

[9] X. Cai and G. B. Giannakis, "Error Probability Minimizing Pilots for OFDM With M-PSK Modulation Over Rayleigh-Fading Channels," IEEE Trans. Veh. Technol., vol. 53, no. 1, pp. 146-155, Jan. 2004.

[10] J. G. Proakis, Digital Communications, 4th ed. New York: McGrawHill, 2001.

[11] A. Ekbal, K.-B. Song, and J. M. Cioffi, "Outage Capacity and Cutoff Rate of Bit-Interleaved Coded OFDM under Quasi-State Frequency Selective Fading," in Proc. IEEE GLOBECOM 2003, vol. 2, Dec. 2003 , pp. $1054-1058$.

[12] J. Wu, C. Xiao, and N. C. Beaulieu, "Optimal Diversity Combining Based on Noisy Channel Estimation," in Proc. IEEE ICC 2004, vol. 1, June 2004, pp. 214-218. 